\title{
A new batch verification scheme for ECDSA* signatures
}

\author{
APURVA S KITTUR* and ALWYN R PAIS \\ Information Security Research Lab, National Institute of Technology Karnataka, Surathkal 575025, India \\ e-mail: apurva.kittur@gmail.com; alwyn.pais@gmail.com
}

MS received 28 December 2018; revised 4 April 2019; accepted 8 April 2019; published online 7 June 2019

\begin{abstract}
In this paper, we propose an efficient batch verification algorithm for ECDSA* (Elliptic Curve Digital Signature Algorithm)* signatures. Our scheme is efficient for both single and multiple signers. ECDSA* signature is a modified version of ECDSA, which accelerates the verification of ECDSA signature by more than $40 \%$. However, the highlighting feature of our proposed scheme is its efficiency for varied batch sizes. The scheme is resistant to forgery attacks by either signer or intruder. The performance of our scheme remains consistent for higher batch sizes too $(\geq 8)$. Our paper also discusses the possible attacks on ECDSA signatures and also how our scheme is resistant to such attacks.
\end{abstract}

Keywords. Digital signatures; batch verification; elliptic Curve Cryptography (ECC); scalar multiplication.

\section{Introduction}

Digital signatures are used to verify the content of the received message and signer's identity in a communication. There are various digital signature verification schemes available for verifying the authenticity of signatures. Some of the schemes are RSA (Rivest, Shamir and Adleman), DSS (Digital Signature Scheme) and Elliptic Curve Digital Signature Algorithm (ECDSA). In recent times ECDSA has gained popularity because of its small public key size, which reduces the burden of computation at the verifier. Therefore ECDSA is considered as one of the lightweight authentication schemes.

Elliptic Curve Cryptography (ECC) is a popularly used standard cryptographic protocol. ECDSA is a digital signature scheme of ECC that is similar to ElGamal signature schemes. ECDSA is also popular because of its small key size and signature size. As we know, most of the ElGamal signature schemes have the property where verification takes more time than signature generation. This property holds true for ECDSA scheme too. Hence, verifying such signatures together in a batch reduces the overhead of sequential verification.

Batch verification is verifying multiple signatures at once signed by either single or multiple signers. For example, there are multiple batch verification schemes available for just RSA digital signature scheme. Hence multiple digital signatures are verified at one instance in a single verification test. Hence it has the advantage of reduced computation time and load at the verifier without compromise in security. Verifying multiple ECDSA signatures in batches

*For correspondence makes the verification more applicable in various real-time applications since it reduces the verification time and CPU consumption.

Batch verification is not a new concept since it has been in use extensively for various signature schemes like RSA, DSS [1-4], etc. The concept was introduced by Fiat [5] and was first successfully implemented by Harn [6] for RSA digital signatures. This technique successfully identifies the existence of an invalid signature in a given batch of RSA signatures. There are various batch verification schemes introduced for verifying ECDSA signatures too. [7, 8] have some of the popular schemes for ECDSA signatures. Even though the existing techniques try to reduce the computation time, most of them are not efficient for larger batch sizes [9].

In modern day applications such as E-cash $[10,11]$, E-voting, Intelligent cars, WSN and IoT network [12-14], etc., it is very important to verify multiple signatures at any given point of time in order to get real-time results. Even though techniques exist to batch verify multiple ECDSA signatures, many of them suffer from one major drawback, i.e., they are not efficient for larger batch sizes. Therefore in real-time scenarios, where a large number of signatures have to verified at once, it is important to have a scheme that performs well irrespective of the batch size without compromise in the security.

In this article, we propose a batch verification scheme for multiple ECDSA* signatures, which is secure in comparison with the existing schemes. ECDSA* signature is a modified version of ECDSA. ECDSA* signature is as secure as ECDSA with reduced computation at the verification. The only disadvantage of ECDSA* signatures is their increased signature size. We have implemented our 
scheme for ECDSA* signatures. The primary advantage of the proposed scheme is that the efficiency of the scheme does not vary significantly with increase in batch size. The other highlighting factor of the proposed scheme is the inclusion of random pairwise co-primes, which enhances the security of our scheme further. We provide the details of our scheme in the later sections.

The major contributions of the paper are as follows:

- Propose a new batch verification scheme for ECDSA* signatures, which is efficient for various batch sizes.

- Detailed security analysis of the proposed scheme is provided.

- Cost and complexity analysis of the proposed scheme is provided.

The paper is organized as follows. In section 2 we discuss the related work. Section 3 provides a brief on ECDSA and ECDSA* $^{*}$ signatures and section 4 gives details of our proposed scheme. Section 5 provides the results and also a comparison to existing techniques, followed by conclusion in section 6.

\section{Related work}

There are several batch verification schemes introduced for batch verification of ECDSA signatures [9]. The technique ECDSA $^{*}$ [7] is introduced to speedup the verification of individual ECDSA signature. The schemes S1 and S2 [8] are very efficient for smaller batch sizes. However, the efficiency of these techniques decreases as the batch size increases. Therefore there is a need for a batch verification technique that performs consistently irrespective of the batch. [15] introduced randomized batch verification.

The ECDSA*[7] signature scheme is a variation of ECDSA scheme. The signature of ECDSA* scheme is $(R, s)$ whereas the original ECDSA signature is $(r, s)$ with respect to $(m, Q)$, where $m$ is the message to be signed and $Q$ is the public key. The ECDSA* scheme involves appending one or more bits of side information to the signature by signer. This increases the size of signature but also reduces the verification time at the verifier by $40 \%$.

The scheme introduced by [16] involves computation of $w$ - Non-Adjacent Forms (NAF) where $w$ is the window width. In this scheme, the square root computation happens by the $w$-NAF method. This scheme is efficient in computing the point $R$ of signature, as compared with traditional approaches.

The other algorithms S1, S2 [8] concentrate on reducing the time required to calculate R.y. Usually, R.y is calculated by square root method in ECDSA. These two techniques introduce different methods to arrive at $R$ to reduce the time taken by square root method.
Table 1. Notations followed in the paper.

\begin{tabular}{lc}
\hline Symbol & Reference to \\
\hline$p$ & order of the prime field $\mathbb{F}_{p}$ \\
$E\left(\mathbb{F}_{p}\right)$ & elliptic curve defined over prime field $\mathbb{F}_{p}$ \\
$P$ & random base point of order $n$ in $E\left(\mathbb{F}_{p}\right)$ \\
$n$ & prime order of $P$ \\
$h(m)$ & hash value of the message $m$ \\
$(m, r, s)$ & signature of ECDSA \\
$(m, R, s)$ & signature of ECDSA* \\
$t$ & batch size of the signatures \\
$h$ & the cofactor $\frac{\left|E\left(\mathbb{F}_{p}\right)\right|}{n}$ \\
\hline
\end{tabular}

\section{Brief introduction to ECDSA signatures}

Koblitz [17] and Miller [18] independently introduced Elliptic Curve Cryptography, which is currently the most secure public key cryptosystem. Elliptic Curve Digital Signature Scheme (ECDSA) is based on Discrete Logarithm Problem (DLP). All the values used for computation in ECDSA belong to the Elliptic Curve group (Table 1).

We make an assumption that $E\left(\mathbb{F}_{p}\right)$ is a cyclic group with $P$ as generator and the cofactor $h=1$; such curves are mostly suggested by NIST. According to Hasse's theorem, we have $|n-p-1| \leq 2 \sqrt{p}$. If $n<p$, then there are maximum two possible representations of an element of $\mathbb{Z}_{n}$ in $\mathbb{Z}_{p}$ and if $n \geq p$, then there is exactly one representation for $\mathbb{Z}_{n}$ in $\mathbb{Z}_{p}$. Therefore, the number of elements in $\mathbb{Z}_{n}$ having two representations is $\leq 2 / \sqrt{p}$.

The ECDSA signature is represented as $(m, r, s)$, where $r$ and $s$ are represented in terms of modulo $n ; m$ is the message that is signed.

\subsection{The ECDSA algorithm}

We provide the algorithms for ECDSA Key-pair Generation (Algorithm 1), Signature Generation (Algorithm 2) and Signature Verification (Algorithm 3). These algorithms explain how an individual ECDSA signature is generated and verified.

\footnotetext{
Algorithm 1 ECDSA Key Generation Algorithm

Input: Standard domain parameters

Output: Key Pairs: Public Key $-Q$, Private Key $-d$

1. For an elliptic curve $E\left(\mathbb{F}_{p}\right)$, choose $P$ of order $n, P \in$ $E\left(\mathbb{F}_{p}\right)$

2. Choose a random integer $d$ such that $2 \leq d \leq n-2$.

3. Compute $Q=d P$.
} 

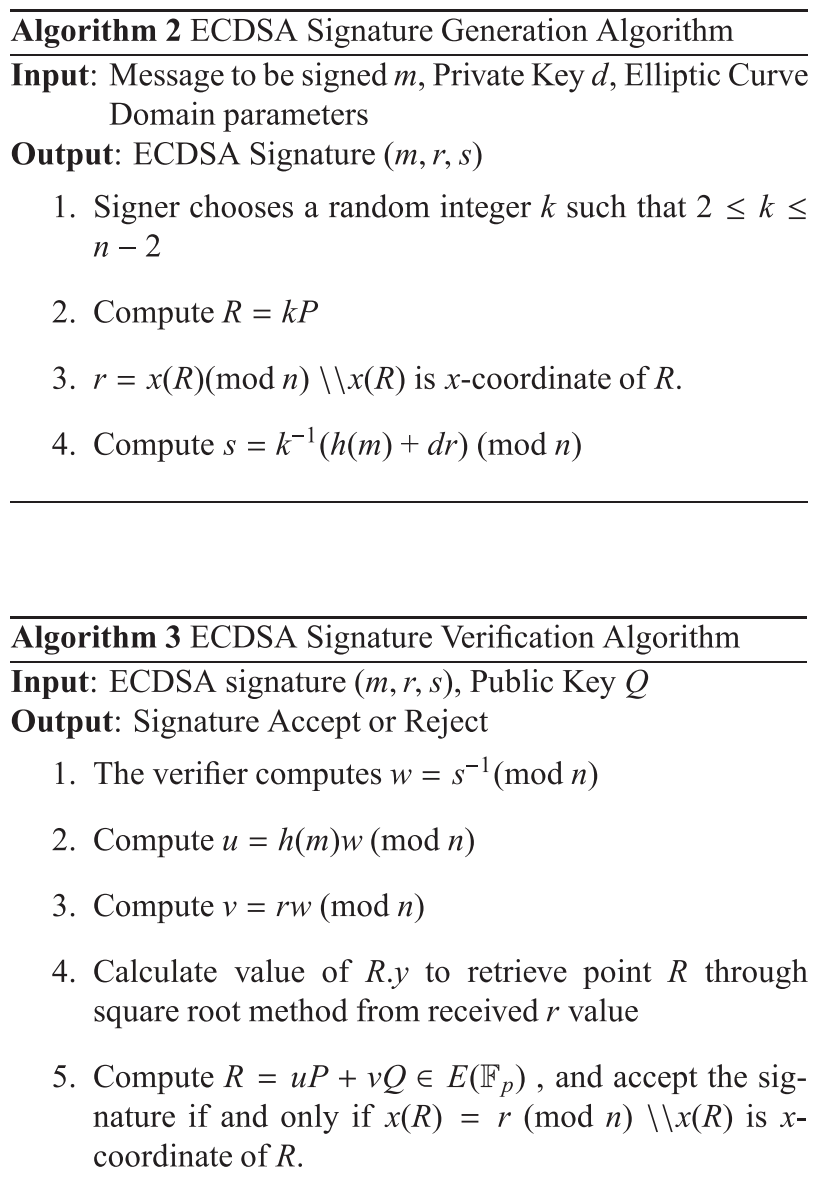

\subsection{The ECDSA* algorithm}

ECDSA* is a modified version of ECDSA algorithm. It has been introduced by Antipa et al [7] and provides $40 \%$ more efficiency in the verification time compared with ECDSA signatures. The only disadvantage of the scheme is its increased signature size. In applications such as IoT where there is a need for quick verification without compromise in security to get results in real-time environment, this signature scheme proves to be efficient. The signature of modified ECDSA* signature scheme is $(R, s)$ whereas the original ECDSA signature is $(r, s)$ with respect to $(m, Q)$, where $r=f(R), m$ is the message to be signed and $Q$ is the public key.

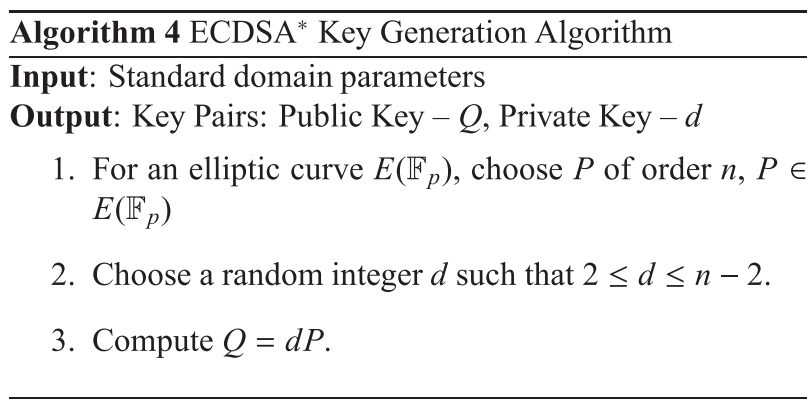

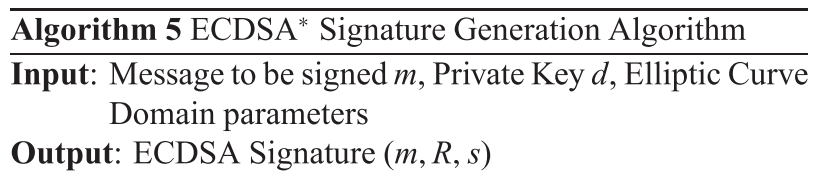

1. Signer chooses a random integer $k$ such that $2 \leq k \leq$ $n-2$

2. Compute $R=k P$

3. $r=x(R)(\bmod n) \backslash \backslash x(R)$ is $x$-coordinate of $R$.

4. Compute $s=k^{-1}(h(m)+d r)(\bmod n)$

\subsection{Batch verification}

As we know, batch verification is verifying multiple signatures at once. The authors of [8] discussed the naive batch verification of ECDSA* signatures. However, the naive batch verification scheme is vulnerable to forgery attacks as described in sections 4.1 and 4.2 of our paper. The standard approach to verify multiple ECDSA and ECDSA $^{*}$ signatures generated by either single or multiple signers is shown in Eqs. (1) and (2).

In case of a single signer

$$
\sum_{i=1}^{t} R_{i}=\left(\sum_{i=1}^{t} u_{i}\right) P+\left(\sum_{i=1}^{t} v_{i}\right) Q .
$$

For multiple signers

$$
\sum_{i=1}^{t} R_{i}=\left(\sum_{i=1}^{t} u_{i}\right) P+\sum_{i=1}^{t} v_{i} Q_{i} .
$$

The standard equation for batch verification of ECDSA* signatures is the same as for ECDSA signatures. The difference between the two lies only in the signature size. Equations (1) and (2) verify $t$ ECDSA or ECDSA* signatures together in a batch, where $i \in 1,2, \ldots, t$. 


\subsection{Threat model}

The attack model is rewritten as follows: an unauthorized third party attacker is the main threat to various digital signature schemes. We have considered various levels of threats depending on the parameters exposed to the attacker.

- Category 1: The attacker has access to a few messagesignature pairs. Based on the same, the attacker tries to forge signatures that can pass the batch verification test.

- Category 2: The attacker gets access to the messagesignature pairs as well as public-private key pairs. Hence he tries to forge signatures that can pass the batch verification test.

- Category 3: The sender himself is compromised and sends illegitimate signature to the verifier, where the signature passes the batch verification as well as individual verification tests. This is a major threat that the verifier has to successfully tackle.

The results in section 5 also show the same for various curves.

\section{Our verification scheme}

The existing batch verification schemes are efficient for signatures of both single and multiple signers. Although the existing batch verification schemes are secure and efficient, the efficiency ceases as the size of the batch increases. In real-time scenarios, the batch size is relatively high and the existing schemes will not be suitable. Our scheme performs better for various batch sizes at the price of a few modular and scalar multiplication operations.

When multiple ECDSA* signatures are generated, the verifier performs batch verification to verify these signatures at once. In our scheme, the verifier generates the pairwise relatively prime equation randomizers $b_{1}, b_{2}, \ldots, b_{t}<n, \in \mathbb{F}_{p}$ during verification; the $b_{1}, b_{2}, \ldots, b_{t}$ are 32-bit numbers. One of the main reasons for using pairwise random numbers and not just random numbers is that random numbers are always odd numbers except 2 . Hence the probability of guessing the random number by the attacker is increased by more than $50 \%$. The pairwise random numbers have even numbers as well. Hence, to improve the security of the scheme further, we have used pairwise random numbers rather than just random numbers.

The pairwise relative primes are the ones where every pair of numbers are relatively prime with each other, i.e., the GCD of every pair of numbers is one. We have generated the relative prime numbers $b_{1}, b_{2}, \ldots, b_{t}$ by considering a set $C$ of first $g$ primes. Let $f(g)$ be such that
$S_{g}^{f(g)}$ (Sterling numbers of second kind) is maximal. For computing $b_{1}, b_{2}, \ldots, b_{f(g)}$, the set of primes $C$ has to be randomly partitioned into $f(C)$ classes $C_{1}, C_{2}, \ldots, C_{f(C)}$, and the elements in each $C_{i}$ are inter-multiplied to obtain $b_{i}$.

This can be explained with an example as follows. Consider the set of first 7 primes $C=\{2,3,5,7,11,13,17\}$. To find relative primes, create two subsets and assign the primes randomly from set $C$ to these subsets: $C_{1}=$ $\{2,5,11\}$ and $C_{2}=\{3,17\}$ and $C_{3}=\{7,13\}$. Now by inter-multiplying any number of elements within $C_{i}$, we obtain $b_{i}$. Therefore $b_{1}=\{2 \times 11\}, b_{2}=\{17\}, b_{3}=\{7 \times$ $13\}$ and so on. Hence if only three $b$ 's are needed, then $b_{1}=22, \quad b_{2}=17$ and $b_{3}=91$. The three numbers $\left\{b_{1}, b_{2}, b_{3}\right\}=\{22,17,91\}$ are relatively prime with each other. If we pick any two numbers of the three, their GCD will be one. For our scheme, we generate the co-primes of 32-bit size.

For the given standard elliptic curve parameters $[a, b, p$, base point $P, n]$, our scheme generates pairwise relative prime randomizers in the range $[1, \ldots, n]$. The reason for implementing this concept on ECDSA* is because in ECDSA, $y$-coordinates of $R_{i}$ in the LHS of Eqs. (3) and (4) are unknown. However, in ECDSA* ${ }^{*} y$-coordinates of value $R_{i}$ are known; hence, using Elliptic Curve scalar multiplication, we can compute LHS and verify the signatures.
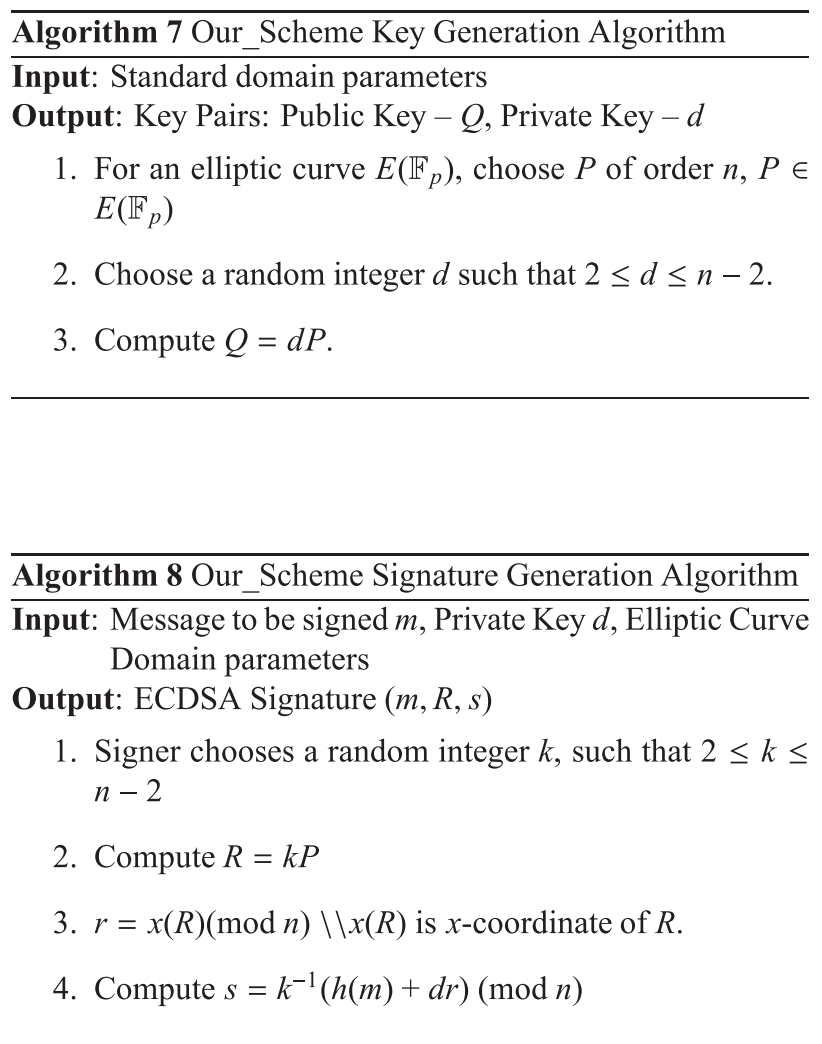

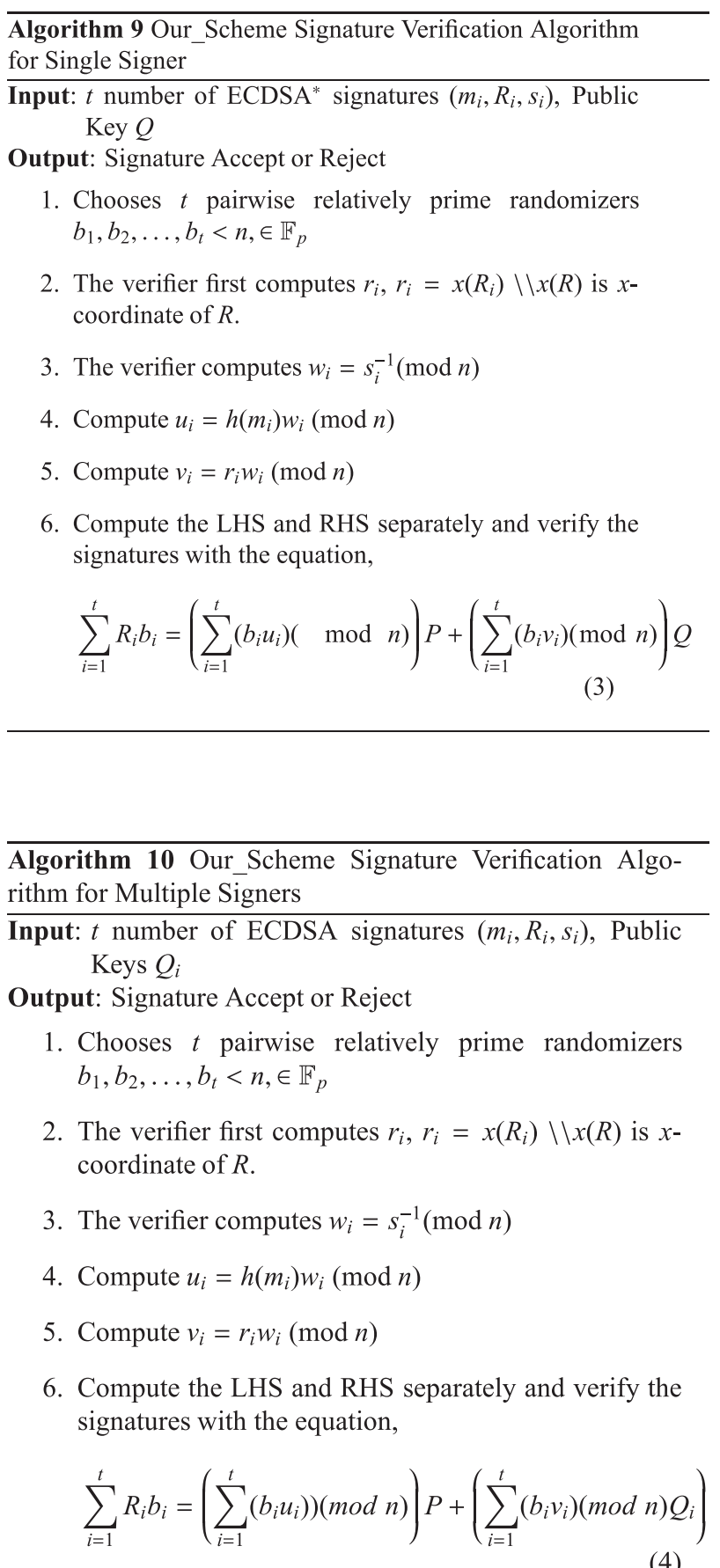

Our scheme is implemented for ECDSA* signatures so as to reduce the overhead of calculating R.y. Our proposed scheme can also be implemented for ECDSA signatures similar to [15], but as the batch size increases, the scheme becomes almost difficult to implement. The four algorithms (7-10) explain our scheme for single and multiple signers.

Hence with ECDSA* signatures, the overhead of computing $R$ is reduced at the verifier by signer sending directly the entire $R$ in the signature along with $s$, instead of $R . x$. Therefore, one major step of calculating $R . y$ through square roots calculation method is reduced. In the Results section, we provide results of our scheme implemented over ECDSA* signatures.

According to the results demonstrated in [7], implementing ECDSA* signature scheme accelerates the verification by $40 \%$. Therefore from algorithm 6 for signature verification, we can observe that amount of time spent in calculating the $R . y$ for every signature is reduced during verification with a minor increase in the signature size. Hence performing the proposed batch verification of such signatures will further reduce the verification time, making the verification scheme more efficient in real-time applications.

\subsection{Security analysis}

In ECDSA* algorithm, the signature $\left(m_{i}, R_{i}, s_{i}\right)$ is generated by the signer. The proposed scheme is a batch verification scheme for ECDSA* signatures. Our scheme is different from the naive batch verification scheme for ECDSA* signatures only in verification procedure.

For the ECDSA* signature scheme, the entire $R_{i}$ is part of the signature generated by the signer, which reduces the time of reconstruction of $y_{i}$ at the verifier. However, this exposes entire $R_{i}$ to the adversary. Hence disclosing $R_{1}, R_{2}, \ldots, R_{t}$ will expose both $x_{i}$ and $y_{i}$ coordinates of every $R_{i}$, which makes the naive batch verification scheme for ECDSA* signatures vulnerable to attacks. Hence introducing $t$ pairwise relatively prime equation randomizers makes such forgery attacks infeasible. The attacker finds it very difficult to guess the random numbers. An $l$-bit randomizer can enhance security by $2^{l}$.

To further enhance security of batch verification scheme, the proposed scheme uses randomizers $b_{1}, b_{2}, \ldots, b_{t}$ that are pairwise relative co-primes. Hence, the proposed scheme provides more security than the schemes that use prime random numbers. We have also presented a forgery attack later on the schemes that use only random prime numbers during batch verification. Even though the proposed batch verification scheme is experimented for ECDSA $^{*}$ signatures, the scheme can also be applied to ECDSA signatures too, since both the schemes are equally secure [7].

\subsection{Possible attacks on the existing schemes}

1. In the attack stated by Bernstein et al [19], an attacker generates two forged signatures among the $t$ signatures. Therefore the verifier has only $t-2$ genuine signatures. If the two signatures are $(r, s)$ and $(r,-s)$ for the same message $m$, generated with the same key pair $(d, Q)$, then the naive batch verification algorithm verifies and accepts all the $t$ signatures. This attack on naive ECDSA and ECDSA* is successful when all the other signatures are from the same signer. 
To overcome such an attack, our scheme generates random pairwise primes during verification. Therefore it becomes very difficult for the attacker to guess the number generated at the verifier. For an $l$-bit relative prime, it takes around $2^{l}$ tests to arrive at the number, which is practically impossible to guess. Hence our scheme can tackle this attack since the attacker cannot guess the random number generated by the verifier during verification.

2. In the second attack, the attacker has the knowledge of valid key pair $\left(d_{1}, Q_{1}\right)$. The attacker tries to forge the signature for message $m_{2}$ by generating $\left(r_{2}, s_{2}\right)$ under any valid key $Q_{2}$ along with the key $Q_{1}$ for message $m_{1}$. The values $r_{2}$ and $s_{2}$ can be found as $R_{2}=k_{2} P$ and $r_{2}=x\left(R_{2}\right)$ with random $s_{2}$. Now for another message $m_{1}, R_{1}$ is computed as $R_{1}=r_{2} s_{2}^{-1} Q_{2} \quad$ and $\quad r_{1}=x\left(R_{1}\right) \quad$ and $s_{1}=\left(h\left(m_{1}\right)+r_{1} d_{1}\right)\left(k_{2}-h\left(m_{2}\right) s_{2}^{-1}\right)^{-1}$. During batch verification using the naive method, $R_{1}+R_{2}=$ $\left(h\left(m_{1}\right) s_{1}^{-1}+h\left(m_{2}\right) s_{2}^{-1}\right) P+r_{1} s_{1}^{-1} Q_{1}+r_{2} s_{2}^{-1} Q_{2}$, which is the same as $\left(k_{2} P+r_{2} s_{2}^{-1} Q_{2}\right)$. Therefore these signatures can be verified as valid during the naive batch verification. In this attack, the attacker tries to forge the signatures and pass the verification test. However, he/she fails to guess the relative prime number generated during verification for every signature. Hence the forger has to guess this number of $l$ bits length from $2^{l}$ possibilities for every signature, which is practically impossible. In our scheme, we use 32-bit co-prime numbers, which challenge the attacker to try all $2^{32}$ possibilities to dig the number up. Therefore our verification scheme ceases the possibility of accepting these invalid signatures.

3. In the third attack, we explain the possibility of an attack if we use the random prime numbers instead of relative pairwise primes for verifying ECDSA* signatures.

Theorem 1 The following statements are equivalent:

(a) there is an efficient batch verification algorithm $\operatorname{Batch}\left(m_{1}, m_{2}, b, b_{1}, b_{2}, P, Q\right)=\left(R_{1}, R_{2}, s_{1}, s_{2}\right)$ for verifying ECDSA* signatures (where $m_{1}, m_{2}$ are the messages and $b, b_{1}, b_{2}$ are randomly generated primes) such that

$$
\begin{aligned}
R_{1} b & +R_{2} b_{i} \\
& =\left(b s_{1}^{-1} h\left(m_{1}\right)+b_{i} s_{2}^{-1} h\left(m_{2}\right)\right) \bmod n \quad P \\
& +\left(b s_{1}^{-1} r_{1}+b_{i} s_{2}^{-1} r_{2}\right) \bmod n \quad Q
\end{aligned}
$$

where $b \neq b_{1} \neq b_{2}$;

(b) an algorithm exists to break this batch verification test.

Proof In order to prove that $a \Rightarrow b$, one has to choose $b, b_{1}, b_{2}$ in such a way that $b^{\prime}=b_{1}-b_{2}$ has an inverse modulo $n$ and compute $\operatorname{Batch}\left(m_{1}, m_{2}, b, b_{1}, b_{2}, p, Q\right)=$ $\left(r_{1}, r_{2}, s_{1}, s_{2}\right)$

$$
\begin{aligned}
R_{1} b+R_{2} b_{i} & =\left(b s_{1}^{-1} h\left(m_{1}\right)+b_{i} s_{2}^{-1} h\left(m_{2}\right)\right) \bmod n \quad P \\
& +\left(b s_{1}^{-1} r_{1}+b_{i} s_{2}^{-1} r_{2}\right) \quad \bmod n \quad Q .
\end{aligned}
$$

Now by subtracting the equations for $i=1,2$, we get

$$
\begin{aligned}
& \left(R_{1} b+R_{2} b_{1}\right)-\left(R_{1} b+R_{2} b_{2}\right) \\
& =\left(\left(b s_{1}^{-1} h\left(m_{1}\right)+b_{1} s_{2}^{-1} h\left(m_{2}\right)\right) \bmod \quad n \quad P\right. \\
& \left.+\left(b s_{1}^{-1} r_{1}+b_{1} s_{2}^{-1} r_{2}\right) \quad \bmod \quad n \quad Q\right) \\
& -\left(\left(b s_{1}^{-1} h\left(m_{1}\right)+b_{2} s_{2}^{-1} h\left(m_{2}\right)\right) \bmod \quad n \quad P\right. \\
& \left.+\left(b s_{1}^{-1} r_{1}+b_{2} s_{2}^{-1} r_{2}\right) \quad \bmod n \quad Q\right) \text {, } \\
& R_{2} b^{\prime}=\left(b^{\prime} s_{2}^{-1} h\left(m_{2}\right)\right) \bmod \quad n \quad P+\left(b^{\prime} s_{2}^{-1} r_{2}\right) \quad \bmod \quad n \quad Q .
\end{aligned}
$$

Now by taking $b^{\text {th }}$ roots $\bmod n$ on both the sides, since $b^{\prime}$ has an inverse modulo $n$, the signature $\left(m_{2}, r_{2}, s_{2}\right)$ passes the batch verification and individual ECDSA* verification tests.

Therefore a lemma can be stated as the immediate consequence to Theorem 4.2.

Lemma 4.2.1 If all the $b_{i}$ are picked from a set $M$, all are pairwise relatively prime and are smaller than $n$ then

$$
\begin{aligned}
& \Omega(M)=\operatorname{Pr}\left[\operatorname{BATCH}\left(b_{i} \in M,\left\{\{r, s\},\left\{r^{\prime}, s^{\prime}\right\}\right\}\right)\right. \\
& \left.\quad=\text { True } \mid \operatorname{ECDSA}(\{\mathrm{r}, \mathrm{s}\}) \wedge \operatorname{ECDSA}\left(\left\{\mathrm{r}^{\prime}, \mathrm{s}^{\prime}\right\}\right)=\text { False }\right] \\
& \quad=\frac{1}{|M|^{2}-|M|} \cong|M|^{-2}
\end{aligned}
$$

Here the most natural set $M$ has all the primes that are less than a certain bound. To optimize the cardinality of $M$, lets us denote $C$ as a set of first $m$ primes and let $f(m)$ be such that $S_{m}^{f(m)}$ is maximal. For computing $b_{1}, b_{2}, \ldots, b_{f(m)}$, $C$ has to be randomly partitioned into $f(m)$ classes $C_{1}, C_{2}, \ldots, C_{f(m)}$, and the elements in each $C_{i}$ are intermultiplied to obtain $b_{i}$. This generates pairwise numbers from an optimized set $C$.

From Lemma 4.2.1, it is clear that we can reduce cardinality of $M$ and still manage to generate more than the cardinality of $M$ relative co-prime numbers. That is, if the cardinality of $M$ is $m$, then we can still generate more than $m$ pairwise prime numbers. This is the reason behind considering pairwise co-prime random numbers rather than random primes. If only primes are considered, then the size of $M$ has to be very large because the number of primes in $2^{16}$ is 6542 , which can be easily guessed by modern computing machines. Hence we generate pairwise co-primes, to restrict the cardinality of $M$ and generate more $b$ 's. This also has an added advantage in security. If the numbers are only prime numbers, then more than $50 \%$ of the computation at the attacker is reduced, since all the even and composite numbers are eliminated. 


\subsection{Run-time analysis comparison}

For Algorithm S1, as stated in [8], the runtime of S1 is $\mathrm{O}\left(\mathrm{m}^{3} t\right)$. The running time analysis states that the algorithm outperforms Naïve batch verification for ECDSA, only in situations where $\sigma$ is rather large compared with $m$, where $\sigma$ is the number of square root operations, and $m$ is $2^{t}$, where $t$ is the batch size. This happens typically when the batch size $t$ is small and the field size $q$ is large. The operations needed for retrieving the values of $y_{i}$ are time consuming and also the linearized systems are solved, where the runtime increases exponentially with the increase in batch size.

For Algorithm S2, as stated in [8], the running time is a little better than that of $\mathrm{S} 1, \mathrm{O}\left(m^{2} t\right)$. It follows that this reconstruction procedure is practical in all cases where running the algorithms Naïve(ECDSA) and S1 is practical. In order to reduce the computation complexity of finding $y_{i}$, the algorithm increases the polynomial arithmetic operations as well as field operations. The algorithm also needs more storage to store the polynomial.

The proposed scheme does not include the square root operation to arrive at the $y_{i}$ co-ordinate of the $R_{i}$ point. The running time of our scheme is $\mathrm{O}(t+s)$, where $t$ is the batch size and $s$ is the number of scalar multiplications. Hence, our scheme does not involve solving the square roots and other polynomial arithmetic operations. Therefore the scheme performs better than the schemes S1 and S2.

\section{Results}

In this section, we provide the results of implementing our batch verification scheme for ECDSA* signatures. We also provide the results of existing schemes and make a comparative study. All the parameters considered for experimentation are standard parameters suggested by NIST (National Institute of Standards and Technology).

We applied our batch verification scheme on three NIST prime curves: $(P-192),(P-224)$ and $(P-256)$ curves. We have analysed the runtime values, the speedup values of all the three curves. We also provide the speedup and verification time values for multiple signers as well a single singer, for all the curves.

The experimentation is carried out on a Rock cluster CentOS 6.0 system. The processor is Intel ${ }^{\circledR}$ Xeon ${ }^{\circledR}$ E52650. Each machine has 20 cores and each core runs on a 2.3-GHz processor. We have also used the CryptoPP library for generating the ECDSA parameters and also for generating the relative prime numbers.

We have compared the runtime of various schemes in this section. The columns indicate the following.

- Batch size: indicates the number of signatures in a batch.

- Individual: these values indicate the time of execution if the signatures are verified individually.

- Naive: this indicates the time taken by the naive batch verification scheme for ECDSA signatures.

- ECDSA*: it indicates the execution time taken by the naive batch verification scheme for ECDSA* signatures.

- S1: the execution time for Algorithm S1 for ECDSA signatures.

- S2: the execution time for Algorithm S2 for ECDSA signatures.

- Our_scheme: the execution time taken by our proposed batch verification scheme for ECDSA* signatures.

In our results for multiple signers, for all the three curves, tables 5, 9 and 13 have two ideal speedup columns. The first column corresponds to the ideal speedup values for the existing techniques, which can be computed as $\frac{2 t}{t+1}$. The second ideal column in these tables represents the ideal expected values for our scheme. These values are computed as $\frac{3 t}{2 t+1}$. The speedup values indicate the performance gain in verification time compared with individual verification.

Tables 2-5 provide results for curve $(P-192)$. Table 2 provides the running time for various batch verification schemes along with our scheme. We can observe from table 2 that our scheme performs better than the individual and the other batch verification schemes except for

Table 2. Verification time for the curve $(P-192)$ for a single signer (s).

\begin{tabular}{|c|c|c|c|c|c|c|}
\hline Batch size & Individual & Naive & $E C D S A^{*}$ & S1 & $S 2$ & Our_scheme \\
\hline 2 & 0.108 & 0.056 & 0.056 & 0.056 & 0.055 & 0.057 \\
\hline 3 & 0.498 & 0.184 & 0.175 & 0.191 & 0.18 & 0.182 \\
\hline 4 & 0.709 & 0.214 & 0.184 & 0.275 & 0.214 & 0.198 \\
\hline 5 & 0.896 & 0.256 & 0.194 & 0.605 & 0.297 & 0.25 \\
\hline 6 & 1.163 & 0.298 & 0.213 & 2.326 & 0.546 & 0.302 \\
\hline 7 & 1.626 & 0.351 & 0.258 & 6.775 & 4.065 & 0.354 \\
\hline 8 & 2.213 & 0.427 & 0.313 & - & - & 0.517 \\
\hline 16 & 9.201 & 0.892 & 0.661 & - & - & 0.963 \\
\hline 32 & 28.64 & 1.429 & 1.108 & - & - & 1.844 \\
\hline 64 & 78.32 & 2.728 & 2.255 & - & - & 3.341 \\
\hline
\end{tabular}


Table 3. Speedup for the curve $(P-192)$ for a single signer.

\begin{tabular}{|c|c|c|c|c|c|c|}
\hline Batch size & Ideal & Naive & $E C D S A^{*}$ & $S 1$ & $S 2$ & Our_scheme \\
\hline 2 & 2 & 1.92 & 1.92 & 1.92 & 1.96 & 1.89 \\
\hline 3 & 3 & 2.70 & 2.84 & 2.6 & 2.76 & 2.73 \\
\hline 4 & 4 & 3.3 & 3.85 & 2.57 & 3.31 & 3.58 \\
\hline 5 & 5 & 3.5 & 4.61 & 1.48 & 3.01 & 3.5 \\
\hline 6 & 6 & 3.9 & 5.45 & 0.50 & 2.13 & 3.85 \\
\hline 7 & 7 & 4.62 & 6.3 & 0.24 & 0.4 & 4.5 \\
\hline 8 & 8 & 5.18 & 7.06 & - & - & 4.28 \\
\hline 16 & 16 & 10.3 & 13.9 & - & - & 9.55 \\
\hline 32 & 32 & 20.04 & 25.8 & - & - & 15.53 \\
\hline 64 & 64 & 28.7 & 34.73 & - & - & 23.44 \\
\hline
\end{tabular}

Table 4. Verification time for the curve (P-192) for multiple signers (s).

\begin{tabular}{|c|c|c|c|c|c|c|}
\hline Batch size & Individual & Naive & $E C D S A^{*}$ & S1 & $S 2$ & Our_scheme \\
\hline 2 & 0.108 & 0.08 & 0.083 & 0.087 & 0.08 & 0.09 \\
\hline 3 & 0.498 & 0.35 & 0.339 & 0.356 & 0.35 & 0.389 \\
\hline 4 & 0.709 & 0.477 & 0.472 & 0.545 & 0.51 & 0.537 \\
\hline 5 & 0.896 & 0.618 & 0.56 & 0.985 & 0.64 & 0.66 \\
\hline 6 & 1.163 & 0.868 & 0.705 & 2.91 & 0.99 & 0.843 \\
\hline 7 & 1.626 & 1.355 & 0.945 & 5.42 & 1.827 & 1.169 \\
\hline 8 & 2.213 & 1.75 & 1.28 & - & - & 1.574 \\
\hline 16 & 9.201 & 7.25 & 5.11 & - & - & 6.35 \\
\hline 32 & 28.64 & 21.12 & 15.31 & - & - & 19.48 \\
\hline 64 & 78.32 & 55.23 & 41.22 & - & - & 52.8 \\
\hline
\end{tabular}

ECDSA* $^{*}$ and naive signature schemes. The reason for this lower performance of our scheme in comparison with these schemes is the introduction of new relative prime integers during verification of ECDSA* signatures. The overhead in our scheme is due to these numbers and increase in the number of modular and scalar multiplication operations. Our scheme performs better than S1 and S2 for all batch sizes except 2 . For larger batch sizes $(\geq 7)$, the performance of algorithms S1 and S2 ceases to decrease drastically and becomes incomparable. However, our scheme maintains its performance for varied batch sizes as compared with S1 and S2 and is more secure compared with all other existing schemes.

We can observe that our scheme performs better than Algorithms S1 and S2 for a single signer. Note that our scheme is fast, scalable and secure among most of the available schemes. We can observe the speedup gained by various schemes in table 3 , where ideal speedup is $t$. The speedup values are computed with respect to the individual running time values. Tables 4 and 5 show an analysis of these schemes for multiple signers. The ideal speedup of our scheme is different from that of other schemes because of the inclusion of extra relative primes.

Table 5. Speedup for the curve $(P-192)$ for multiple signers.

\begin{tabular}{cccccccc}
\hline Batch size & Ideal & Naive & ECDSA & S1 & S2 & Ideal & Our_scheme \\
\hline 2 & $\mathbf{1 . 3 3}$ & 1.28 & 1.30 & 1.23 & 1.34 & $\mathbf{1 . 2}$ & 1.17 \\
3 & $\mathbf{1 . 5 0}$ & 1.42 & 1.47 & 1.4 & 1.41 & $\mathbf{1 . 2 8 6}$ \\
4 & $\mathbf{1 . 6 0}$ & 1.5 & 1.50 & 1.30 & 1.39 & $\mathbf{1 . 3 3 4}$ \\
5 & $\mathbf{1 . 6 7}$ & 1.45 & 1.60 & 0.91 & 1.4 & $\mathbf{1 . 3 6 3}$ \\
6 & $\mathbf{1 . 7 1}$ & 1.34 & 1.65 & 0.4 & 1.17 & $\mathbf{1 . 3 8 5}$ \\
7 & $\mathbf{1 . 7 5}$ & 1.20 & 1.72 & 0.30 & 0.89 & $\mathbf{1 . 4}$ \\
8 & $\mathbf{1 . 7 8}$ & 1.26 & 1.73 & - & - & $\mathbf{1 . 4 1 2}$ \\
16 & $\mathbf{1 . 8 8}$ & 1.27 & 1.80 & - & - & $\mathbf{1 . 4 5 4}$ \\
32 & $\mathbf{1 . 9 4}$ & 1.35 & 1.87 & - & - & $\mathbf{1 . 4 7 7}$ \\
64 & $\mathbf{1 . 9 7}$ & 1.41 & 1.9 & - & - & $\mathbf{1 . 4 8 3}$ \\
\hline
\end{tabular}


Table 6. Verification time for the curve $(P-224)$ for a single signer (s).

\begin{tabular}{|c|c|c|c|c|c|c|}
\hline Batch size & Individual & Naive & $E C D S A^{*}$ & $S 1$ & $S 2$ & Our_scheme \\
\hline 2 & 0.1575 & 0.091 & 0.090 & 0.081 & 0.08 & 0.091 \\
\hline 3 & 0.280 & 0.118 & 0.113 & 0.104 & 0.113 & 0.098 \\
\hline 4 & 0.412 & 0.144 & 0.135 & 0.146 & 0.120 & 0.140 \\
\hline 5 & 0.536 & 0.180 & 0.145 & 0.319 & 0.161 & 0.151 \\
\hline 6 & 0.732 & 0.242 & 0.178 & 1.22 & 0.293 & 0.193 \\
\hline 7 & 0.888 & 0.256 & 0.191 & 4.44 & 0.986 & 0.203 \\
\hline 8 & 1.067 & 0.273 & 0.212 & - & - & 0.235 \\
\hline 16 & 2.74 & 0.403 & 0.333 & - & - & 0.421 \\
\hline 32 & 8.94 & 0.789 & 0.663 & - & - & 0.803 \\
\hline 64 & 32.11 & 1.5408 & 1.284 & - & - & 1.596 \\
\hline
\end{tabular}

Table 7. Speedup for the curve $(P-224)$ for a single signer.

\begin{tabular}{|c|c|c|c|c|c|c|}
\hline Batch size & Ideal & Naive & $E C D S A^{*}$ & S1 & $S 2$ & Our_scheme \\
\hline 2 & 2 & 1.73 & 1.75 & 1.95 & 1.98 & 1.73 \\
\hline 3 & 3 & 2.37 & 2.47 & 2.69 & 2.82 & 2.85 \\
\hline 4 & 4 & 2.85 & 3.05 & 2.82 & 3.43 & 2.94 \\
\hline 5 & 5 & 2.97 & 3.69 & 1.68 & 3.3 & 3.55 \\
\hline 6 & 6 & 3.02 & 4.11 & 0.6 & 2.5 & 3.79 \\
\hline 7 & 7 & 3.47 & 4.64 & 0.2 & 0.9 & 4.37 \\
\hline 8 & 8 & 3.91 & 5.03 & - & - & 4.54 \\
\hline 16 & 16 & 6.8 & 8.23 & - & - & 6.5 \\
\hline 32 & 32 & 11.3 & 13.48 & - & - & 11.1 \\
\hline 64 & 64 & 20.8 & 25 & - & - & 20.12 \\
\hline
\end{tabular}

Table 6 shows the results obtained from curve $(P-224)$. The parameters considered for experimentation of these algorithms are the standard values suggested by NIST. Table 7 shows the speedup for a single signer. The speedup includes both scalar multiplication and batch verification time. The maximum speedup is obtained for ECDSA* signatures, followed by the naive verification scheme and our scheme. Tables 8 and 9 show the results for multiple signers.

The results for curve $(P-256)$ are also similar to those of the other curves. The running time and speedup values for single and multiple signers are depicted in tables 10-13. The relative performance of the schemes remains the same.

\subsection{Computation cost analysis}

In this subsection, we analyse the computational cost of various ECC operations obtained during our experimentation. The proposed batch verification scheme is very similar to the standard batch verification scheme for ECDSA* with difference in extra computation of random co prime numbers. We explain these computations in detail one by one.

We will analyse the number of various operations in standard batch verification for ECDSA* for a given batch of $t$ signatures.

Batch verification algorithm for ECDSA signatures has various time-consuming operations such as point addition and scalar multiplication. These operations affect the

Table 8. Verification time for the curve $(P-224)$ for multiple signers (s).

\begin{tabular}{|c|c|c|c|c|c|c|}
\hline Batch size & Individual & Naive & $E C D S A^{*}$ & $S 1$ & $S 2$ & Our_scheme \\
\hline 2 & 0.1575 & 0.13 & 0.127 & 0.122 & 0.12 & 0.135 \\
\hline 3 & 0.280 & 0.21 & 0.210 & 0.2 & 0.195 & 0.221 \\
\hline 4 & 0.412 & 0.307 & 0.290 & 0.31 & 0.275 & 0.311 \\
\hline 5 & 0.536 & 0.412 & 0.37 & 0.531 & 0.383 & 0.398 \\
\hline 6 & 0.732 & 0.567 & 0.47 & 1.49 & 0.653 & 0.536 \\
\hline 7 & 0.888 & 0.632 & 0.562 & 2.69 & 1.03 & 0.645 \\
\hline 8 & 1.067 & 0.751 & 0.667 & - & - & 0.768 \\
\hline 16 & 2.74 & 1.879 & 1.62 & - & - & 1.925 \\
\hline 32 & 8.94 & 5.87 & 5.02 & - & - & 6.144 \\
\hline 64 & 32.11 & 20.73 & 17.54 & - & - & 21.81 \\
\hline
\end{tabular}


Table 9. Speedup for the curve $(P-224)$ for multiple signers.

\begin{tabular}{cccccccc}
\hline Batch size & Ideal & Naive & ECDSA & S1 & S2 & Ideal \\
\hline 2 & $\mathbf{1 . 3 3}$ & 1.21 & 1.24 & 1.29 & 1.32 & $\mathbf{1 . 2}$ & Our_scheme \\
3 & $\mathbf{1 . 5 0}$ & 1.34 & 1.33 & 1.40 & 1.43 & $\mathbf{1 . 2 8 6}$ \\
4 & $\mathbf{1 . 6 0}$ & 1.34 & 1.42 & 1.33 & 1.50 & $\mathbf{1 . 3 3 4}$ \\
5 & $\mathbf{1 . 6 7}$ & 1.30 & 1.45 & 1.01 & 1.4 & $\mathbf{1 . 3 6 3}$ \\
6 & $\mathbf{1 . 7 1}$ & 1.29 & 1.55 & 0.49 & 1.13 & $\mathbf{1 . 3 8 5}$ \\
7 & $\mathbf{1 . 7 5}$ & 1.4 & 1.58 & 0.33 & 0.86 & $\mathbf{1 . 4}$ \\
8 & $\mathbf{1 . 7 8}$ & 1.42 & 1.6 & - & - & $\mathbf{1 . 4 1 2}$ \\
16 & $\mathbf{1 . 8 8}$ & 1.45 & 1.69 & - & - & $\mathbf{1 . 4 5 4}$ \\
32 & $\mathbf{1 . 9 4}$ & 1.52 & 1.78 & - & - & $\mathbf{1 . 4 7 7}$ \\
64 & $\mathbf{1 . 9 7}$ & 1.55 & 1.83 & - & $\mathbf{1 . 4 8 8}$ \\
\hline
\end{tabular}

Table 10. Verification time for the curve $(P-256)$ for a single signer (s).

\begin{tabular}{ccccccc}
\hline Batch size & Individual & Naive & ECDSA & S1 & S2 & Our_scheme \\
\hline 2 & 0.153 & 0.079 & 0.079 & 0.078 & 0.077 & 0.097 \\
3 & 0.276 & 0.102 & 0.096 & 0.105 & 0.13 & 0.102 \\
4 & 0.457 & 0.135 & 0.12 & 0.154 & 0.19 & 0.307 \\
5 & 0.663 & 0.173 & 0.14 & 1.209 & 1.271 \\
6 & 0.866 & 0.240 & 0.162 & 2.876 & - & 0.157 \\
7 & 1.093 & 0.225 & 0.172 & - & - & 0.201 \\
8 & 1.385 & 0.238 & 0.186 & - & - & 0.431 \\
16 & 4.737 & 0.408 & 0.332 & - & - \\
32 & 18.703 & 0.758 & 0.627 & 1.275 & -615 \\
64 & 76.462 & 1.52 & & -630 \\
\hline
\end{tabular}

Table 11. Speedup for the curve $(P-256)$ for a single signer.

\begin{tabular}{ccccccc}
\hline Batch size & Ideal & Naive & ECDSA $^{*}$ & S1 & S2 & Our_scheme \\
\hline 2 & 2 & 1.94 & 1.94 & 1.96 & 1.98 & 1.92 \\
3 & 3 & 2.70 & 2.87 & 2.62 & 2.84 & 2.7 \\
4 & 4 & 3.38 & 3.81 & 2.97 & 3.5 & 3.48 \\
5 & 5 & 3.83 & 4.73 & 1.96 & 3.48 & 4.22 \\
6 & 6 & 3.6 & 5.34 & 0.71 & 2.82 & 4.63 \\
7 & 7 & 4.86 & 6.35 & 0.38 & 0.86 & 5.44 \\
8 & 8 & 5.82 & 7.44 & - & - & 6.21 \\
16 & 16 & 11.6 & 14.27 & - & - & 10.99 \\
32 & 32 & 24.67 & 29.83 & - & - & 22.95 \\
64 & 64 & 50.09 & 59.97 & - & - & 46.9 \\
\hline
\end{tabular}

verification time of signatures and also help in analysing and comparing the efficiency of the various schemes. Tables 14 and 15 provide the number of operations required for verification using standard batch verification scheme and our proposed scheme. In table 14, we can observe that our proposed scheme has relatively higher number of scalar and modular multiplications for single signer as compared with the standard batch versification scheme. Similarly, 15 represents the operation-count of batch verification schemes for signatures signed by multiple signers.

Table 12. Verification time for the curve $(P-256)$ for multiple signers (s).

\begin{tabular}{|c|c|c|c|c|c|c|}
\hline Batch size & Individual & Naive & $E C D S A^{*}$ & $S 1$ & $S 2$ & Our_scheme \\
\hline 2 & 0.153 & 0.116 & 0.116 & 0.116 & 0.115 & 0.128 \\
\hline 3 & 0.276 & 0.194 & 0.19 & 0.194 & 0.189 & 0.215 \\
\hline 4 & 0.457 & 0.30 & 0.295 & 0.319 & 0.305 & 0.344 \\
\hline 5 & 0.663 & 0.44 & 0.404 & 0.619 & 0.44 & 0.487 \\
\hline 6 & 0.866 & 0.61 & 0.52 & 1.574 & 0.66 & 0.627 \\
\hline 7 & 1.093 & 0.761 & 0.63 & 3.036 & 1.23 & 0.786 \\
\hline 8 & 1.385 & 0.969 & 0.819 & - & - & 0.988 \\
\hline 16 & 4.737 & 3.025 & 2.513 & - & - & 3.273 \\
\hline 32 & 18.703 & 11.82 & 10.33 & - & - & 12.80 \\
\hline 64 & 76.462 & 49.04 & 40.456 & - & - & 51.63 \\
\hline
\end{tabular}


Table 13. Speedup for the curve $(P-256)$ for multiple signers.

\begin{tabular}{cccccccc}
\hline Batch size & Ideal & Naive & ECDSA & S1 & S2 & Ideal & Our_scheme \\
\hline 2 & $\mathbf{1 . 3 3}$ & 1.32 & 1.31 & 1.31 & 1.32 & $\mathbf{1 . 2}$ & 1.19 \\
3 & $\mathbf{1 . 5 0}$ & 1.42 & 1.45 & 1.42 & 1.46 & $\mathbf{1 . 2 8 6}$ & 1.281 \\
4 & $\mathbf{1 . 6 0}$ & 1.50 & 1.55 & 1.43 & 1.5 & $\mathbf{1 . 3 3 4}$ & 1.329 \\
5 & $\mathbf{1 . 6 7}$ & 1.51 & 1.64 & 1.07 & 1.49 & $\mathbf{1 . 3 6 3}$ \\
6 & $\mathbf{1 . 7 1}$ & 1.42 & 1.67 & 0.55 & 1.31 & $\mathbf{1 . 3 8 5}$ \\
7 & $\mathbf{1 . 7 5}$ & 1.43 & 1.72 & 0.36 & 1.89 & $\mathbf{1 . 4}$ & 1.380 \\
8 & $\mathbf{1 . 7 8}$ & 1.43 & 1.69 & - & - & $\mathbf{1 . 4 1 2}$ & 1.39 \\
16 & $\mathbf{1 . 8 8}$ & 1.566 & 1.74 & - & - & $\mathbf{1 . 4 5 4}$ \\
32 & $\mathbf{1 . 9 4}$ & 1.58 & 1.81 & - & - & $\mathbf{1 . 4 7 7}$ \\
64 & $\mathbf{1 . 9 7}$ & 1.56 & 1.89 & - & $\mathbf{1 . 4 8 8}$ \\
\hline
\end{tabular}

Table 14. Various operations of batch verification for a single signer.

\begin{tabular}{lcc}
\hline & \multicolumn{2}{c}{ No. of computations } \\
\cline { 2 - 3 } Operation & ECDSA* & Our_scheme \\
\hline Point addition & $t$ & $t$ \\
Scalar multiplication & 2 & $t+2$ \\
Modular multiplication & $2 t$ & $4 t$ \\
\hline
\end{tabular}

Table 15. Various operations of batch verification for multiple signers.

\begin{tabular}{lcc}
\hline \multirow{2}{*}{ Operation } & \multicolumn{2}{c}{ No. of computations } \\
\cline { 2 - 3 } & ECDSA* & Our_scheme \\
\hline Point addition & $2 t-1$ & $2 t-1$ \\
Scalar multiplication & $t+1$ & $2 t+1$ \\
Modular multiplication & $2 t$ & $4 t$ \\
\hline
\end{tabular}

Table 16. Execution time for expensive operations.

\begin{tabular}{lc}
\hline Operation & Time (s) \\
\hline Point addition & $2.193 \times 10^{-5}$ \\
Scalar multiplication & $3.24 \times 10^{-3}$ \\
\hline
\end{tabular}

Now we analyse the time taken by the two computationexpensive operations: point addition and scalar multiplication.

From table 16, we can observe that scalar multiplication takes more computation time than point addition. Even though our scheme has greater number of scalar multiplications, it is resistant against forgery attacks whereas the standard batch verification scheme is not. However, the number of point addition operations remains the same for both the schemes. Hence the overhead incurred is minimum compared with the security it provides.

\subsection{Running time analysis}

The existing schemes S1 and S2 have various running time complexities. Most of the time is consumed in solving the multivariate equations to arrive at the value of R.y. Therefore, the running time of Algorithm S1 as stated in [8] is $\Theta\left(m^{3}\right)$ where $m=2^{t}$ and $t$ is the batch size. The running time of the naive scheme involves computation of $t$ modular square roots in $\mathbb{F}_{p}$. If we assume the time for each square root computation as $\sigma$, then the complexity can be stated as $\mathrm{O}((\sigma+m) t)$. We can clearly observe that Algorithm $\mathrm{S} 1$ can perform better than naive algorithm only if $(\sigma+m) t \gg m^{3}$ and it happens only if the batch size is small, i.e., if $t<6$.

Similarly Algorithm S2 has the running time complexity of $\mathrm{O}\left(m t^{2}\right)$, which is better than $\Theta\left(m^{3}\right)$ for Algorithm S1. Therefore $(\sigma+m) t \gg O\left(m t^{2}\right)$ condition is not always true in all cases. For smaller batch sizes, Algorithm S2 performs better than naive algorithm whereas $(\sigma+m) t \gg m^{3}$ is true in most of the cases.

We analysed that the running time of naive, S1 and S2 schemes depends on recreating the point $R$ from $R . x$ and finding the value of $R . y$. In our scheme, we have implemented the algorithm on ECDSA* where there is increase in the signature size, which significantly reduces the computation of R.y. Hence the performance of our scheme is similar to that of the naive scheme. The naive scheme has overhead because of square root calculation and our scheme has overhead in generating the pairwise random prime numbers $b_{i}$. Hence their speedup almost goes hand in hand. The complexity of generating these primes is $\mathrm{O}(t * \log \log t)$.

\section{Conclusion}

Batch verification is a very important concept that is used for verifying multiple signatures at once to reduce computation load and time. In today's world, it is important to have batch verification schemes that are secure as well as efficient for a wide range of batch sizes. From the results 
obtained, it is clear that our technique is efficient for a wide range of batch sizes of ECDSA* signatures with security level better than the existing ones. In real-time applications, it is very important to have verification schemes that are fast as well as secure in verification of signatures. Therefore, our scheme is implemented over ECDSA* signatures. Hence, our technique will be more suitable for real-time applications. Our future plan is to simulate some real-time application using our batch verification technique.

\section{Acknowledgements}

The authors would like to thank Ministry of Electronics \& Information Technology (MeitY), Government of India, for their support in a part of the research.

\section{References}

[1] Lim C H and Lee P J 1994 Security of interactive DSA batch verification. Electron. Lett. 30: 1592-1592

[2] Bao F, Lee C C and Hwang M S 2006 Cryptanalysis and improvement on batch verifying multiple RSA digital signatures. Appl. Math. Comput. 172: 1195-1200

[3] Kittur A S, Jain A and Pais A R 2017 Fast verification of digital signatures in IoT. In: Proceedings of the International Symposium on Security in Computing and Communication. Springer, Singapore, pp. 16-27.

[4] Naccache D, M'Ralhi D, Vaudenay S and Raphaeli D 1994 Can DSA be improved? Complexity trade-offs with the digital signature standard. In: Proceedings of the Workshop on the Theory and Application of Cryptographic Techniques, pp. 77-85

[5] Fiat A 1997 Batch RSA. J. Cryptol. 10: 75-88

[6] Harn L 1998 Batch verifying multiple RSA digital signatures. Electron. Lett. 34: 1219-1220

[7] Antipa A, Brown D, Gallant R, Lambert R, Struik R and Vanstone S 2005 Accelerated verification of ECDSA signatures. In: Proceedings of the International Workshop on Selected Areas in Cryptography. Berlin-Heidelberg: Springer, LNCS vol. 3897, pp. 307-318
[8] Karati S, Das A, Roychowdhury D, Bellur B, Bhattacharya D and Iyer A 2014b New algorithms for batch verification of standard ECDSA signatures. J. Cryptogr. Eng. 4: 237-258

[9] Kittur A S and Pais A R 2017 Batch verification of digital signatures: approaches and challenges. J. Inf. Secur. Appl. 37: $15-27$

[10] Furnell S M and Karweni T 1991 Security implications of electronic commerce: a survey of consumers and businesses. Internet Res. 9: 372-382

[11] Claessens J, Dem V, De Cock D, Preneel B and Vandewalle J 2002 On the security of today's online electronic banking systems. Comput. Secur. 21: 253-265

[12] Zhou L, Schneider F B and Van Renesse R 2002 COCA: a secure distributed online certification authority. ACM Trans. Comput. Syst. 20: 329-368

[13] Zhang Z K, Cho M C Y, Wang C W, Hsu C W, Chen C K and Shieh S 2014 IoT security: ongoing challenges and research opportunities. In: Proceedings of the 7th IEEE International Conference on Service-Oriented Computing and Applications (SOCA), pp. 230-234

[14] Suo H, Wan J, Zou C and Liu J 2012 Security in the internet of things: a review. In: Proceedings of the International Conference on Computer Science and Electronics Engineering (ICCSEE), vol. 3, pp. 648-651

[15] Karati S, Das A and Roychoudhury D 2014 Randomized batch verification of standard ECDSA signatures. In: Proceedings of the International Conference on Security, Privacy, and Applied Cryptography Engineering, pp. 237255

[16] Cheon J H and Yi J H 2007 Fast batch verification of multiple signatures. In: Proceedings of the International Workshop on Public Key Cryptography. Berlin-Heidelberg: Springer, pp. 442-457

[17] Koblitz N 1987 Elliptic curve cryptosystems. Math. Comput. 48: 203-209

[18] Miller V S 1985 Use of elliptic curves in cryptography. In: Proceedings of the Conference on the Theory and Application of Cryptographic Techniques, pp. 417-426

[19] Bernstein D J, Doumen J, Lange T and Oosterwijk J J 2012 Faster batch forgery identification. In: Proceedings of the International Conference on Cryptology in India (INDOCRYPT), pp. 454-473 